OPEN ACCESS

Edited by:

Bey Hing Goh,

Monash University Malaysia, Malaysia

Reviewed by:

Pouya Hassandarvish,

University of Malaya, Malaysia

Yin-Quan Tang,

Taylor's University, Malaysia

${ }^{*}$ Correspondence:

Qi Li

Izwf@hotmail.com

Wen-Hai Wang

wang180sg@163.com

Shi-Bing Su

shibingsu07@163.com

${ }^{\dagger}$ These authors share first authorship

Specialty section: This article was submitted to Ethnopharmacology,

a section of the journal

Frontiers in Pharmacology

Received: 06 January 2020 Accepted: 14 January 2021 Published: 22 March 2021

Citation:

Yang $M-D$, Zhou $W$-J, Chen $X-L$, Chen J, Ji Q, Li Q, Wang W-H and Su S-B (2021) Therapeutic Effect and Mechanism of Bushen-Jianpi-Jiedu Decoction Combined with Chemotherapeutic Drugs on Postoperative Colorectal Cancer. Front. Pharmacol. 12:524663. doi: 10.3389/fphar.2021.524663

\section{Therapeutic Effect and Mechanism of Bushen-Jianpi-Jiedu Decoction Combined with Chemotherapeutic Drugs on Postoperative Colorectal Cancer}

\author{
Meng-Die Yang ${ }^{1 \dagger}$, Wen-Jun Zhou ${ }^{1 \dagger}$, Xiao-Le Chen ${ }^{1}$, Jian Chen ${ }^{1,2,3}$, Qing $\mathrm{Ji}^{4}$, Qi Li ${ }^{4 *}$, \\ Wen-Hai Wang ${ }^{5 *}$ and Shi-Bing Su ${ }^{1 *}$
}

${ }^{1}$ Research Center for Traditional Chinese Medicine Complexity System, Institute of Interdisciplinary Integrative Medicine Research, Shanghai University of Traditional Chinese Medicine, Shanghai, China, ${ }^{2}$ Shanghai TCM-Integrated Hospital, Shanghai University of Traditional Chinese Medicine, Shanghai, China, ${ }^{3}$ Department of Vascular Disease, Shanghai TCM-Integrated Institute of Vascular Disease, Shanghai, China, ${ }^{4}$ Department of Oncology, Shuguang Hospital Affiliated to Shanghai University of Traditional Chinese Medicine, Shanghai, China, ${ }^{5}$ Department of Oncology, Shanghai Baoshan Hospital of Integrated Traditional Chinese and Western Medicine, Shanghai, China

There is a lack of effective therapeutic drugs in patients with postoperative colorectal cancer (PCRC). This study aimed to investigate the therapeutic effect and mechanisms of Bushen-Jianpi-Jiedu decoction (BSJPJDD) combined with chemotherapeutic drugs (oxaliplatin) on PCRC with liver and kidney yin deficiency and spleen deficiency syndrome (LKYD-SDS) through the therapeutic evaluation of clinical therapy and the integrative analysis of network pharmacology, RNA-seq and label-free data, and experiment verification in vitro. In clinical therapy, the median progression-free survival (PFS) and Karnofsky performance score (KPS) were increased in PCRC patients by the aqueous extract of BSJPJDD combined with oxaliplatin treatment for three months, compared to oxaliplatin alone $(p<0.05)$. The integrative analysis showed that 559 differentially expressed genes (DEGs) and 11 differentially expressed proteins (DEPs) were regulated by BSJPJDD, among which seven bioactive compounds through 39 potential targets were involved in the regulation of multiple signaling pathways including MAPK, PI3K-Akt, and HIF-1, etc. In the experimental verification, an ELISA assay showed that plasma ZEB2, CAT, and KRT78 were decreased, and IL-1A, CD5L, FBLN5, EGF, and KRT78 were increased in comparison to the above $(p<0.05)$. Furthermore, the SW620 cell viability was inhibited and the expressions of MAPK and the $\mathrm{p}$-ERK/ERK ratio were significantly downregulated by the aqueous extract of BSJPJDD combined with oxaliplatin treatment, compared with oxaliplatin treatment alone $(p<0.05)$. These data suggested that BSJPJDD combined with oxaliplatin prolongs the survival and improves Karnofsky performance status of PCRC patients with LKYD-SDS, and may be associated with the regulation of multiple signaling pathways.

Keywords: BSJPJDD, therapeutic effect, post operation, colorectal cancer, mechanism 


\section{INTRODUCTION}

Colorectal cancer (CRC), one of the most common cancers in the world, has become the third and second leading cause of cancer death among men and women, respectively (Barzi et al., 2017; Chen et al., 2017; Bos et al., 2018). Most patients of CRC are diagnosed at clinical stages II-III (Bos et al., 2018). As the main adjuvant treatment after CRC surgery, chemotherapy still plays a role in CRC treatment (Di Costanzo et al., 2003). However, the use of chemotherapy alone is insufficient, as there are adverse reactions to chemotherapy; the poor tolerance of patients and chemotherapy resistance (Panczyk, 2014; Li et al., 2019), therefore, an adjuvant therapy of postoperative chemotherapy is needed. The efficacy of drugs is usually evaluated by KPS which is a scoring standard for the functional status of the body. The higher the score, the better health condition, and the more the body can tolerate the side effects of treatment with a higher quality of life. It has been reported that traditional Chinese medicine (TCM) combined with chemotherapy can improve CRC treatment, through reducing postoperative ileus and urinary, improving the tolerance of patients and the complications and resistance of chemotherapy, remodeling the gut microbiota and the tumor microenvironment, suppressing thymidylate synthase, and preventing cancer recurrence and metastasis, etc. (Tan et al., 2008; Ye et al., 2015; Liu et al., 2017a; Sui et al., 2017; Liu et al., 2018a; Zhang et al., 2018; Lv et al., 2019; Hong et al., 2020).

In China, TCM is often used for the treatment of CRC ( $\mathrm{Su}$ et al., 2012; Yang et al., 2019). In the clinical practice of TCM, treatment is usually based on TCM syndrome differentiation (Hu et al., 2018). TCM syndrome types in CRC have different metabolic and molecular characteristics (Ji et al., 2017; Wu et al., 2019b), and their treatments are according to the different indications. Previous research has showed that about $12 \%$ of CRC patients have liver and kidney yin deficiency and spleen deficiency syndrome (LKYD-SDS) (Zhang et al., 2013). The clinical manifestation of LKYDSDS can be seen as hypochondriac and costal pain, weakness of the waist and knee, hot waves, night sweats, dry mouth and throat, fatigue, tinnitus, abdominal distention, loose stool, pale or red tongue, little or light fur, and a thin pulse. CRC patients with LKYD-SDS are often treated with the Bushen-Jianpi rule. It also has been reported that Bushen-Jianpi formula (BSJPF) can improve the survival time of liver cancer patients and regulate cell apoptosis in vitro (Cai et al., 2018). Moreover, the Jianpi-Jiedu formula (JPJDF) can improve the survival rate in patients with stage II and III CRC and inhibits tumorigenesis, metastasis, and angiogenesis through the mTOR/HIF-1alpha/VEGF pathway in CRC (Wu et al., 2019a).

The Bushen-Jianpi-Jiedu decoction (BSJPJDD) is one of the Bushen-Jianpi formulae, and consists of eight Chinese herbs. It contains Astragalus membranaceus Fisch. ex Bunge., Rehmannia glutinosa (Gaertn.) DC., Atractylodes macrocephala Koidz., Cornus officinalis Siebold \& Zucc., Dioscorea oppositifolia L., Sophora flavescens Aiton., Vitis quinquangularis Rehd., and
TABLE 1 | The herbal composition of BSJPJDD.

\begin{tabular}{lc}
\hline Herbs & Dose (g) \\
\hline Astragalus membranaceus Fisch. ex Bunge & 18 \\
Rehmannia glutinosa (Gaertn.) DC. & 15 \\
Atractylodes macrocephala Koidz & 15 \\
Cornus officinalis Siebold \& Zucc & 9 \\
Dioscorea oppositifolia L. & 9 \\
Sophora flavescens Aiton & 9 \\
Vitis quinquangularis Rehd & 30 \\
Akebia trifoliata (Thunb.) Koidz & 30
\end{tabular}

Akebia trifoliata (Thunb.) Koidz. However, the effects and molecular mechanisms of the aqueous extract of BSJPJDD combined with chemotherapeutic drugs (oxaliplatin) on CRC with LKYD-SDS are still unclear.

For many years, TCM has relied on the herbal formula, through a synergistic effect of various herbal medicines to achieve attenuation and efficiency in the treatment of complex diseases and syndromes. But it is difficult to analyze the effective mechanism of the formula by traditional pharmacological evaluations. As new fields in TCM studies, network pharmacology, transcriptomics, proteomics, and systems biology can be used for clarifying the effects and molecular mechanisms of TCM with a system perspective (Wu et al., 1997; Shi et al., 2014), it provides an approach for the further investigation of BSJPJDD and improves chemotherapy to treat CRC along with LKYD-SDS.

Therefore, this study investigated the effects and the pharmacological mechanisms of the aqueous extract of BSJPJDD combined with oxaliplatin on postoperative CRC (PCRC) with LKYD-SDS using survival analysis, integrative analysis with RNA-seq and label-free and network pharmacology analysis, and pharmacological experiment verification in vitro. Our study showed that BSJPJDD combined with oxaliplatin prolongs the survival and improves the life quality of PCRC patients with LKYD-SDS, and its pharmacological mechanisms may be involved in the comprehensive regulation of multi-compounds, multi-targets, and multi-pathways.

\section{MATERIALS AND METHODS}

\section{Reagents}

Tetrazolium compound 3-(4,5-dimenthyl-2-yl)-5(3carboxymethoxyphenyl)-2- (4sulfophenyl)-2H-tetrazolium MTS (lot\# 313103) was obtained from Promega (United States). CD 3, CD 4, CD 8, and NK cell assay kits were obtained from $\mathrm{BD}$ Biosciences Pharmingen (United States). Oxaliplatin (bzl800003) was purchased from Aosaikang (Nanjing, China) Antibodies. The primary antibodies against ERK (lot\# 4695), p-ERK (lot\# 4370), p38 (lot\# 9212), p-p38 (lot\# 4511), JNK (lot\# 9252), and p-JNK (lot\# 4668) from Cell Signaling Technology (United States) and the secondary antibody against GAPDH (lot\# AF0911) from Affinity Biosciences (United States) were used. 
TABLE 2 | The inclusion and exclusion criteria of chemotherapy plans.

Serious complications With other serious diseases Incomplete medical records

\section{Bushen-Jianpi-Jiedu Decoction Preparation}

The herbal composition of BSJPJDD is shown in Table 1. All herbs were purchased from ShuGuang Hospital, Shanghai University of TCM and soaked in $2 \mathrm{~L}$ of water for $30 \mathrm{~min}$, boiled for $30 \mathrm{~min}$, then filtered three times. Finally, a concentration of $5.6 \mathrm{~g} / \mathrm{ml}$ of aqueous extracts were made. HPLC-MS MRM chromatograms of the aqueous extracts of BSJPDD are shown in Supplementary Figure S1. The concentration of morroniside and loganin in BSJPJDD were $0.092 \mu \mathrm{g} / \mathrm{ml}$ (Supplementary Figure S1C) and $0.231 \mathrm{mg} / \mathrm{ml}$ (Supplementary Figure S1D), respectively. The reference standard of morroniside and loganin were purchased from the Shanghai Research Center of Chinese Medicine Standardization. The mobile phases comprised eluent A ( $0.1 \%$ formic acid) and eluent $B$ (methanol). The gradient flow was as follows: $0-5 \mathrm{~min}$, $30 \%$ of $\mathrm{B} ; 5-40 \mathrm{~min}, 30-90 \%$ of $\mathrm{B} ; 40-45 \mathrm{~min}, 90-100 \%$ of $\mathrm{B}$; $45-50 \mathrm{~min}, 100 \%$ of $\mathrm{B}$. The analysis was performed at a flow rate of $1.0 \mathrm{ml} / \mathrm{min}$. The injection volume was $10 \mu \mathrm{L}$.

\section{Patient Characteristics and Treatments}

A total of 149 patients with CRC were recruited from March 2014 to February 2018 at the Shanghai University of TCM Shu Guang Hospital. Patients were divided into a BSJPJDD group and oxaliplatin group (control group). The oxaliplatin group was treated following chemotherapy plans including FOLFIRI (Irinotecan $180 \mathrm{mg} / \mathrm{m}^{2}$, Day $1+$ Leucovorin $400 \mathrm{mg} / \mathrm{m}^{2}$, Day $1+$ Fluorouracil $400 \mathrm{mg} / \mathrm{m}^{2}$, Day $1+$ Fluorouracil $2,400 \mathrm{mg} / \mathrm{m}^{2}$ until $48 \mathrm{~h}$ ), XELOX (Oxaliplatin $130 \mathrm{mg} / \mathrm{m}^{2}$, Day $1+$ Xeloda $1,000 \mathrm{mg} / \mathrm{m}^{2}$, bid for 14 days), FOLFOX4 (Oxaliplatin $85 \mathrm{mg} / \mathrm{m}^{2}$,

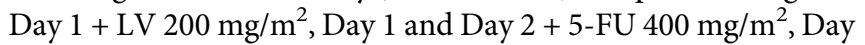
1 and Day 2), and FOLFOX6 (Oxaliplatin $85 \mathrm{mg} / \mathrm{m}^{2}$, Day $1+$ leucovorin $400 \mathrm{mg} / \mathrm{m}^{2}$, Day $1+$ fluorouracil $400 \mathrm{mg} / \mathrm{m}^{2}$, Day $1+$ fluorouracil $2,400 \mathrm{mg} / \mathrm{m}^{2}$ until $48 \mathrm{~h}$ ). The BSJPDD group was treated with the aqueous extract of BSJPDD $(5.6 \mathrm{~g} / \mathrm{ml}$, once a day) based on the treatment of the above chemotherapy plans. The all of patients underwent $\geq 12$ weeks of treatments.

The inclusion criteria and the exclusion criteria are shown in Table 2. Additional information regarding this study includes the following: clinical trials, entitled Study of TCM Syndrome of Hepatocellular Carcinoma and Colorectal Cancer Based on System Science (govIdentifieris NCT03189992), and retrospectively registered in June 16, 2017. All experimental protocols were approved by the IRB of the Shuguang Hospital affiliated with the Shanghai University of Traditional Chinese Medicine (2014-345-41-01) in accordance with the Guidelines for Ethical Review of Drug Clinical Trials, the Declaration of
Helsinki, the International Ethical Guidelines for Biomedical Research Involving Human Subjects (CIOMS: 2002).

\section{Total RNA Extractions and RNA-Seq Analysis}

Total RNA in leukocytes from the PCRC patients with LKYDSDS $(n=12)$, PCRC patients without LKYD-SDS $(n=9)$, the BSJPJDD group before treatment $(n=6)$, and the BSJPJDD group after treatment $(n=6)$ were isolated using TRIzol Reagent (Cat\# 15596-018, Life Technologies, Carlsbad, CA, United States) following the manufacturer's instructions and checked for an RNA integrity number (RIN) to inspect RNA integration using an Agilent Bioanalyzer 2,100 (Agilent Technologies, Santa Clara, CA, United States). Qualified total RNA was further purified using an RNeasy Mini Kit (Cat\# 74106, QIAGEN, GmBH, Germany) and an RNase-Free DNase Set (Cat\# 79254, QIAGEN) and stored at $-80^{\circ} \mathrm{C}$. Quality control was carried out using the RIN number obtained from the Agilent Bioanalyzer 2,100 (Agilent Technologies). An RNA-seq analysis was conducted on each total RNA sample by Illumina RS-122-2401. The data were normalized using Trimmomatic. The differentially expressed genes (DEGs) were screened with fold changes $\geq 1.5$ and $p \leq 0.05$.

\section{Sample Preparation and Label-Free Analysis}

Plasma samples from the PCRC patients with LKYD-SDS $(n=3)$ and PCRC patients without LKYD-SDS $(n=3)$ were ground to a fine powder in liquid nitrogen and suspended in STD buffer $(4 \%$ SDS, $1 \mathrm{mmol} / \mathrm{L}$ DTT, $150 \mathrm{mmol} / \mathrm{L}$ TrisHCl, $\mathrm{pH} 8.0)$. The suspension was vortexed and incubated in boiling water for $5 \mathrm{~min}$. The supernatant was obtained by centrifugation after ultrasonication. The protein concentration of each sample was determined using a 2D Quantification Kit (GE Healthcare, Buckinghamshire, United Kingdom). A total of $100 \mu \mathrm{g}$ of each sample was denatured, reduced, and alkylated as described in the label-free protocol. Each sample was digested overnight with $0.1 \mu \mathrm{g} / \mu \mathrm{L}$ of trypsin solution at $37^{\circ} \mathrm{C}$. The digested peptides were dried by vacuum centrifugation. The nine samples were pooled and vacuum-dried. The pooled sample was separated on a Poly-LC strong cation exchange column $(4.6 \mathrm{~mm} \times 100 \mathrm{~mm})$ using a Nano HPLC System (GE Healthcare). Subsequently, the fractionated samples were analyzed by LC-MS/MS based on a Q-Exactive mass spectrometer (Thermo Finnigan, CA, United States). For peptide data analysis, raw mass data were processed using the Proteomics Tools (Abcam, Cambridge, 
United Kingdom) and normalized according to signal values. The differentially expressed proteins (DEPs) were identified with BRB-array Tools.

Enzyme-Linked Immunosorbent Assay Test Plasma samples of PCRC patients with LKYD-SDS treated with BSJPJDD $(n=58)$ and without BSJPJDD $(n=58)$ were used in the ELISA test. ZEB2, IL-1A, CAT, RECK, LCP1, CD5L, EGF, ALML5, HLA-A, FBLN-5, and KRT78 were tested using ELISA kits according to protocols (ml0202932, Shanghai Enzyme-linked Biotechnology Co., Ltd., Shanghai, China). Briefly, a $50 \mu \mathrm{l}$ serum sample was diluted 1:1, then a $50 \mu \mathrm{l}$ biotin-labeled antibody was added into the reaction hole, incubated at $37^{\circ} \mathrm{C}$ for $1 \mathrm{~h}$, and then $80 \mu \mathrm{l}$ of streptavidin-HRP was added, incubated at $37^{\circ} \mathrm{C}$ for $30 \mathrm{~min}$, then $50 \mu$ of substrates $\mathrm{A}$ and $\mathrm{B}$ were added, incubated at $37^{\circ} \mathrm{C}$ for $10 \mathrm{~min}$. OD value was measured at $450 \mathrm{~nm}$ after adding $50 \mu \mathrm{l}$ of termination solution.

\section{Network Pharmacology Analysis}

The chemical compounds and their targets of BSJPJDD were identified using the TCM database at Taiwan (http://tcm.cmu. edu.tw/), TCM systems pharmacology (TCMSP) database (http:// lsp.nwu.edu.cn/tcmsp.php), and PDTCM (http://cadd.gdhtcm. com:2180/PDTCM/index.php). The compounds were screened for oral bioavailability $(\mathrm{OB})>30 \%$ and drug-likeness $(\mathrm{DL})>0.18$. The targets of PCRC were collected from GeneCards (https:// www.genecards.org/) and the OMIM database (www.omim.org/); the screening criteria were liver cancer, highlights, and scores $>25$. The targets networks of BSJPJDD on CRC were constructed by the Cytoscape software (Version 3.2.0, National Institute of General Medical Sciences, Bethesda, MD, United States). Related parameters were calculated to explore significant nodes (Liu et al., 2017b). An evaluation of the network stabilization was carried out according to Liu et al. (Song et al., 2016). All of the enriched functions and pathways were obtained by OmicsBean (www. omicsbean.cn/).

\section{MTS Assay}

An SW620 cell is a human colorectal adenocarcinoma cell line. It has been chosen in this study according to previous research reports (Hu et al., 2019; Limagne et al., 2019). The SW620 cell line was purchased from the Type Culture Collection of the Chinese Academy of Sciences (Shanghai, China), and the cells were cultured as previously described (Labianca et al., 2010). SW620 cells were cultured in a 96-well plate with a density of $5.0 \times 10^{3}$ cells/well for $24 \mathrm{~h}$, followed by incubation with the aqueous extract of BSJPJDD $(1.56,3.13,6.25,12.5,25,50$, and $100 \mathrm{mg} / \mathrm{ml})$, oxaliplatin $(1.56,3.13,6.25,12.5,25,50$, and $100 \mu \mathrm{g} /$ $\mathrm{ml})$, and BSJPJDD $(3.84$ or $1.92 \mathrm{mg} / \mathrm{ml})$ combined with oxaliplatin $(1.56,3.13,6.25,12.5,25,50$, and $100 \mu \mathrm{g} / \mathrm{ml})$ at $37^{\circ} \mathrm{C}$ for $24 \mathrm{~h}$. Then $20 \mu \mathrm{l}$ of CellTiter AQ solution (G3580, Promega) was added to each well. After $4 \mathrm{~h}$, the absorbance at $490 \mathrm{~nm}$ was measured, and the cell viability was calculated. The combination indices (CIs) were analyzed using the Compusyn software (ComboSyn, Paramus, United States). The CI value $<1$ denotes a synergistic effect, the $\mathrm{CI}$ value $=1$ denotes an additive
TABLE 3 | Characteristics and baseline data of patients with PCRC.

\begin{tabular}{lccc}
\hline Characteristics & $\begin{array}{c}\text { BSJPJDD } \\
(\mathbf{N}=\mathbf{8 3})\end{array}$ & $\begin{array}{c}\text { Control } \\
\mathbf{( N = 6 6 )}\end{array}$ & $\boldsymbol{p}$ \\
& $\mathbf{n}(\%)$ & $\mathbf{n}(\%)$ & \\
\hline Age & $62.85 \pm 9.26$ & $60.95 \pm 9.88$ & 0.137 \\
Gender & & & 0.824 \\
$\quad$ Male & $25(30.12)$ & $21(31.82)$ & \\
Female & $58(69.88)$ & $45(68.18)$ & \\
Pathological differentiation & & & 0.944 \\
$\quad$ Middle-high & $65(78.31)$ & $52(78.79)$ & \\
Low & $18(21.69)$ & $14(21.21)$ & \\
Immune cells marker & & & \\
CD3 (\%) & $61.53 \pm 11.16$ & $63.49 \pm 13.2$ & 0.904 \\
CD4 (\%) & $34.63 \pm 10.07$ & $38.59 \pm 11.36$ & 0.166 \\
CD8 (\%) & $32.2 \pm 11.67$ & $30.18 \pm 10.93$ & 0.440 \\
CD4/CD8 (\%) & $2.2 \pm 5.83$ & $1.54 \pm 0.94$ & 0.403 \\
NK (\%) & $20.85 \pm 12.05$ & $19.98 \pm 10.22$ & 0.742 \\
\hline
\end{tabular}

effect, and the $\mathrm{CI}$ value $>1$ denotes an antagonistic effect (Chou and Talalay, 2010).

\section{Western Blot Assay}

SW620 cells were cultured in a 6-well plate with a density of $1.0 \times$ $10^{6}$ cells/well for $24 \mathrm{~h}$ followed by incubation with $3.84 \mathrm{mg} / \mathrm{mL}$ of BSJPDD extracts and $15.8 \mu \mathrm{g} / \mathrm{ml}$ of oxaliplatin for $48 \mathrm{~h}$. Then, the cells were collected and assayed as described in previous literature. The primary antibodies including ERK, p-ERK, p38, p-p38, JNK, and $p$-JNK (all of 1:1,000) and secondary antibody GAPDH $(1: 5,000)$ were used. Gray value of the Western blot results was calculated by the one-way analysis of variance (ANOVA), performed by Image 1.8.0. $p<0.05$ was considered significant. The assays were repeated at least three times.

\section{Statistical Analysis}

The one-way analysis of variance (ANOVA), rank-sum test, Kaplan-Meier curves, univariate analysis, multivariate Cox regression analysis, and Karnofsky performance score (KPS) analysis were performed (SPSS 18.0 software). $p<0.05$ was considered significant.

\section{RESULTS}

\section{Patient Characteristics, Survival, and Karnofsky Performance Score Analysis}

In this study, 83 (55.7\%) CRC patients with LKYD-SDS were treated with the aqueous extract of BSJPJDD combined with oxaliplatin. The patient characteristics and baseline are listed in Table 3. As shown in Figure 1A, the survival analysis revealed that the median progression-free survival (PFS) of the BSJPJDD group was longer than those of the control group $(p=0.008)$. As shown in Figure 1B, the KPS in advanced stage in the BSJPJDD group was significantly increased compared to the control group $(p<0.05)$. These results indicated that BSJPJDD may increase the 

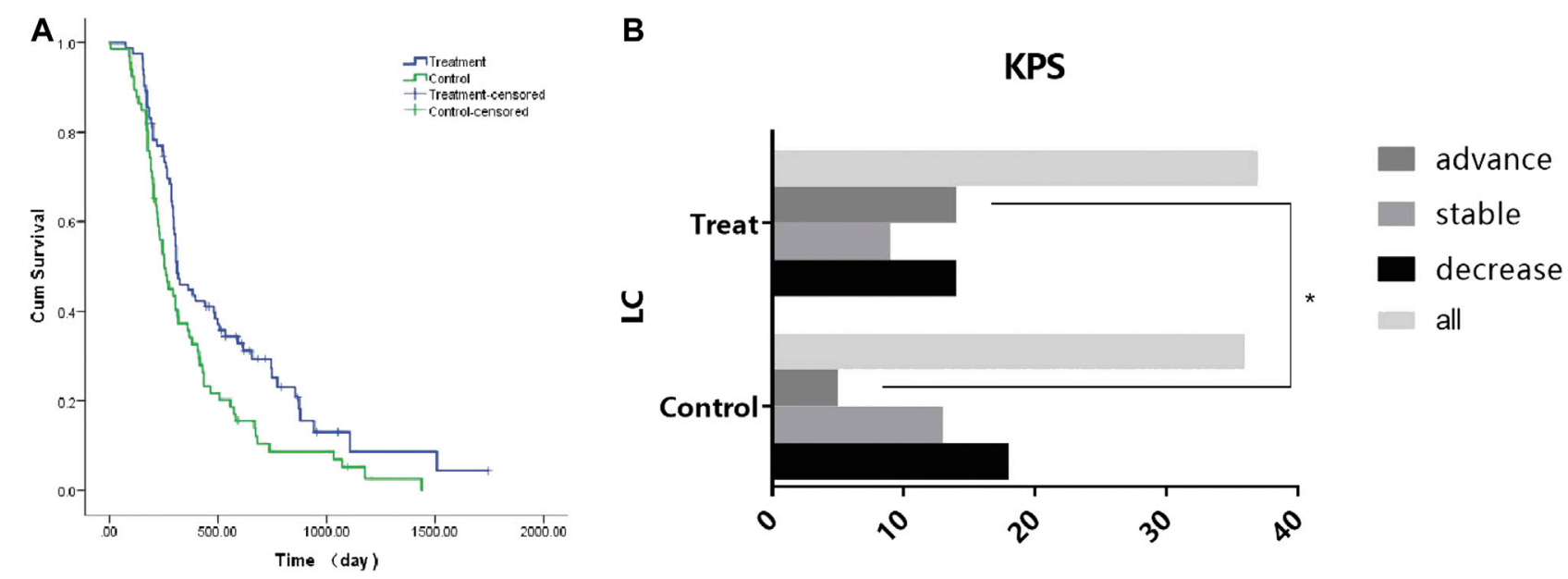

FIGURE 1 | Survival and KPS analysis in CRC treated with BSJPJDD $(\mathrm{n}=3)$ snd without BSJPJDD $(\mathrm{n}=66)$. (A) Survival analysis, (B) KPS * $p<0.05$, BSJPJDD group vs control group.

efficacy of oxaliplatin, improve the survival time and Karnofsky performance status of PCRC patients with LKYD-SDS.

\section{Differentially Expressed Genes and Network Pharmacology Analysis of Bushen-Jianpi-Jiedu Decoction in PCRC Patients with Liver and Kidney Yin Deficiency and Spleen Deficiency Syndrome}

The expressions of mRNA were detected by RNA-seq analysis. A total of 2,845 DEGs in PCRC patients with and without LKYDSDS and 1,573 DEGs in PCRC patients treated with and without BSJPJDD were found. Venn analysis shows that there were 559 crossed DEGs (Figure 2A), indicating that there are DEGs of BSJPJDD in PCRC patients with LKYD-SDS. A BSJPJDD network consisted of 559 DEGs and 11 DEPs. It was constructed to screen the major potential targets of BSJPJDD on PCRC with LKYD-SDS treatment. After a comprehensive evaluation by the topological parameters including topological coefficient, clustering coefficient, degree, closeness centrality, and betweenness centrality, 39 nodes were retained (Figure 2B). Based on these 39 nodes, a targets network of BSJPJDD was constructed, as shown in Figure 2C. Moreover, the major functions and signaling pathways of BSJPJDD on PCRC with LKYD-SDS treatment were found by the enrichment analysis of GO (Figure 3A) and pathway (Figure 3B). It shows that BSJPJDD was involved in the regulation of multiple functions including MAPK cascade, positive regulation of MAP kinase activity, protein binding, Ras guanyl-nucleotide exchange factor activity, regulation of canonical Wnt signaling pathway, etc., and multiple signaling pathways, including MAPK, PI3KAkt, and HIF-1, etc. Furthermore, it was found that seven bioactive compounds (isorhamnetin, kaempferol, 8Isopentenyl-kaempferol, norartocarpetin, wighteone, quercetin, and beta-sitosterol) were found in the BSJPJDD, among which quercetin and wighteone targeted IL-1A regulating P38, JNK, and isorhamnetin, and wighteone and quercetin targeted EGF regulating JNK and ERK in the MAPK pathway (Figure 3C), which further indicated how the bioactive compounds of BSJPJDD are involved in the regulation of the these signal pathways through their targets.

\section{Effects of Bushen-Jianpi-Jiedu Decoction on the Plasma Protein Expressions in PCRC Patients with Liver and Kidney Yin Deficiency and Spleen Deficiency Syndrome}

The expressions of plasma proteins were detected by label-free analysis. Eleven DEPs were found in CRC patients with LKYDSDS treated with and without BSJPJDD. Furthermore, the DEPs were verified by the ELISA assay. As shown in Figure 4, plasma ZEB2, CD5L, FBLN5, and CAT were significantly increased, IL$1 \mathrm{~A}, \mathrm{EGF}$, and KRT78 were significantly decreased in the BSJPJDD group compared to the control group $(p<0.05)$. It indicated that these plasma proteins may be potential indicators for assessing the effects of BSJPJDD on PCRC with LKYD-SDS treatment.

\section{Synergistic Effects of Bushen-Jianpi-Jiedu Decoction Combined with Oxaliplatin on SW620 Cell Viability}

The cell viability was detected by an MTS assay. As shown in Figures 5A,B, the cell viability in SW620 cells were inhibited by the aqueous extract of BSJPJDD ( $\mathrm{IC}_{50}: 3.84 \mathrm{mg} / \mathrm{ml}$ ) and oxaliplatin $\left(\mathrm{IC}_{50}: 15.8 \mu \mathrm{g} / \mathrm{ml}\right)$ treatments in a concentrationdependent manner, respectively. Moreover, the inhibition rate of the SW620 cells in the 3.84 and $1.92 \mathrm{mg} / \mathrm{ml} \mathrm{BSJPJDD-treated}$ groups were higher than that in the oxaliplatin-treated group, and there was a significant difference between the $3.84 \mathrm{mg} / \mathrm{ml}$ 


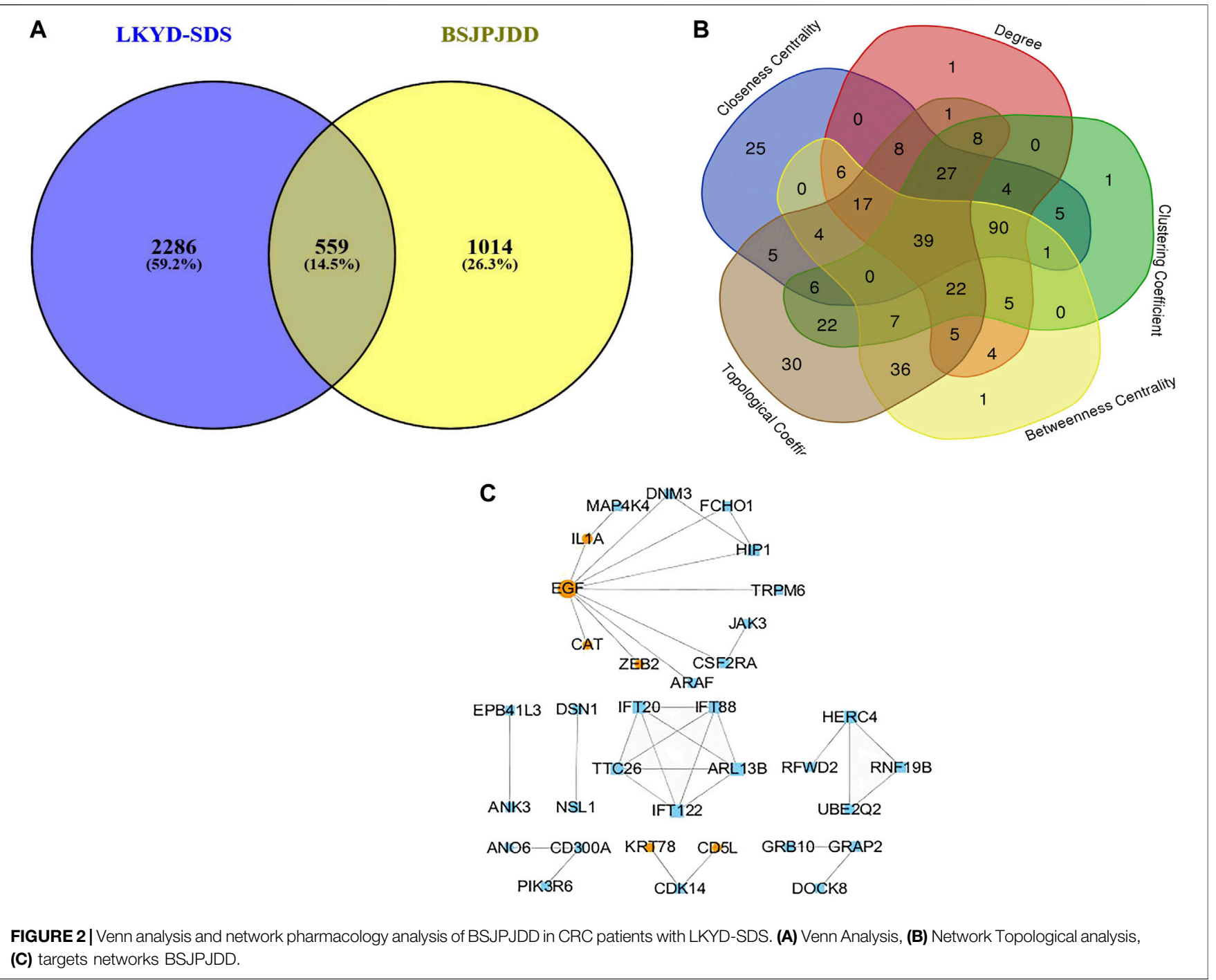

BSJPJDD-treated group and the oxaliplatin-treated group $(p<$ 0.001 ), but not in those of the $1.92 \mathrm{mg} / \mathrm{ml} \mathrm{BSJPJDD}$-treated group $(p>0.05)$ for $24 \mathrm{~h}$ or $48 \mathrm{~h}$ (Figures 5C,D). The CI values of the combination treatment were $1.01,0.72,0.58,0.44,0.28$, and 0.22 , respectively (Figure 5E). It indicated that BSJPJDD combined with oxaliplatin had a synergistic effect that can inhibit the viability of SW620 cells, and that $3.84 \mathrm{mg} / \mathrm{ml}$ of BSJPJDD and $15.8 \mu \mathrm{g} / \mathrm{ml}$ of oxaliplatin were suitable for further experiments of effective mechanisms.

\section{Synergistic Effects of BSJPJDD and Oxaliplatin on the MAPK Pathway in SW620 Cells}

The expressions of ERK, p-ERK, p38, p-p38, JNK, and p-JNK of the MAPK pathway in SW620 cells were detected by Western blot assay. As shown in Figure 6, p-ERK1/2 ratio were significantly upregulated in the combination group (BSJPJDD + oxaliplatin) compared to each single treatment of BSJPJDD, oxaliplatin, or control (no treatment) $(p<0.001$, Figure 6B) and JNK was significantly downregulated ( $p<0.05$, Figure 6F) in BSJPJDD combined with oxaliplatin compared to control alone. ERK1/2, p38, p-p38, and p-JNK had no obvious change (Figures 6C-E, G). These results indicated that the MAPK/ERK pathway was involved in the synergistic effects of BSJPJDD and oxaliplatin on SW620 cell viability.

\section{DISCUSSION}

Clinical relapse is a major reason for postoperative CRC-related deaths, about $33-50 \%$ of CRC patients relapse, and more than $90 \%$ of relapses occur during the first five years following surgery and at a particularly higher rate in the first two years (Binefa et al., 2014), therefore, an effective treatment is extremely important for PCRC. Currently, chemotherapy is still the main strategy for the treatment of PCRC (Li et al., 2013; Huang et al., 2019), which can prolong survival rate and reduce recurrence in patients. However, the tolerance of chemotherapeutic strength and quality of life in cancer patients during chemotherapy are important issues. Even 


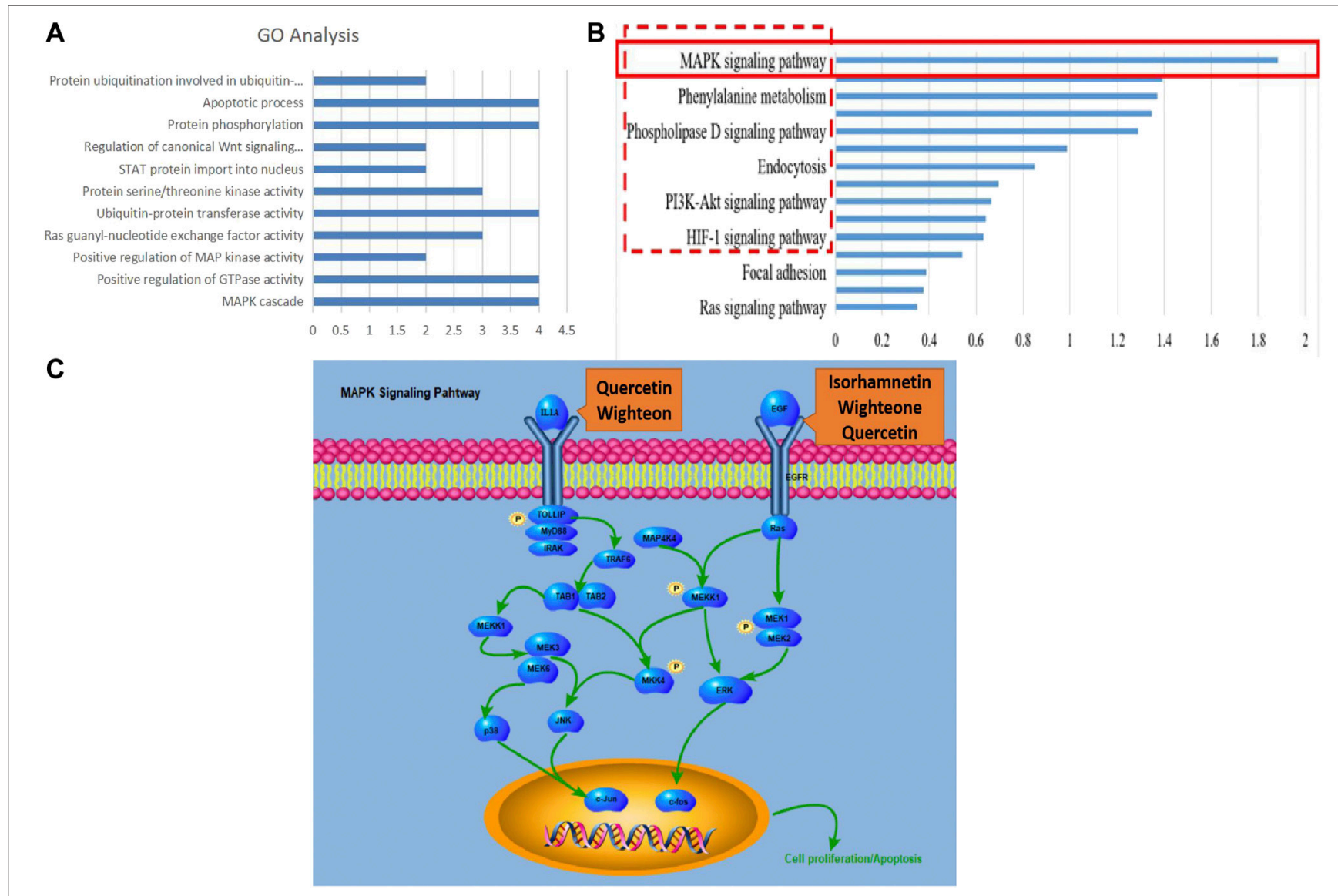

FIGURE 3 | GO and pathway enrichment analysis and components-targets pathways process of BSJPJDD therapy. (A) enriched GO, (B) enriched signaling pathways, (C) components -targets-pathways-process.

though TCM has proven to be an effective therapy in CRC treatments (Zhang et al., 2018; Zhu et al., 2018), the effects and molecular mechanisms of TCM treatment combined with oxaliplatin on PCRC are still unclear.

This study investigates the therapeutic effect and mechanism of the aqueous extract of BSJPJDD combined with oxaliplatin (FOLFIRI, XELOX, FOLFOX4, and FOLFOX6) on PCRC with LKYD-SDS. After treatment for three months, PFS was significantly extended and KPS was significantly increased in PCRC patients treated with the aqueous extract of BSJPJDD combined with oxaliplatin compared with those of oxaliplatin alone (Figure 1). These results suggested that BSJPJDD is beneficial when used with oxaliplatin for PCRC patients with LKYD-SDS. Further research will demonstrate whether BSJPJDD combined with oxaliplatin treatment for 6-12 months is more effective than oxaliplatin alone for PCRC.

The treatment of cancer under the holistic or systemic view of TCM has the characteristics of overall regulation with multimolecular, multi-targets, multi-function, and multi-pathways, which is consistent with the principle of systems biology and network pharmacology. It has been widely applied in effective mechanisms of TCM treatments (Zhang et al., 2013; Cai et al.,
2018; Wu et al., 2019a) and also applied in the research of Chinese herbal formulae on CRC treatment (Liu et al., 2018b; Gong et al., 2019). This study further investigated the effective mechanisms of BSJPJDD by transcriptomics, proteomics, network pharmacology analysis, and experimental verification. A total of 559 DEGs and 11 DEPs were found to be involved in the regulation of BSJPJDD effects on PCRC patients with LKYD-SDS (Figure 2A). Our results (Figure 4) showed that ZEB2, CD5L, FBLN5, CAT, IL-1A, EGF, and KRT78 may be the potential targets involved in the effective mechanisms of the aqueous extract of BSJPJDD combined with oxaliplatin for PCRC with LKYD-SDS treatment.

Moreover, according to the evaluation of primary network nodes and the shared signaling pathways of potential targets of BSJPJDD on PCRC with LKYD-SDS, it was found that BSJPJDD was involved in the regulation of multiple functions including MAPK cascade, positive regulation of MAP kinase activity, protein binding, Ras guanyl-nucleotide exchange factor activity, regulation of the canonical Wnt signaling pathway (Figure 3A), and multiple intracellular signaling, such as the MAPK, PI3K-Akt, and HIF-1 pathways (Figure 3B). Furthermore, seven bioactive compounds (isorhamnetin, kaempferol, 8-Isopentenyl-kaempferol, norartocarpetin, 

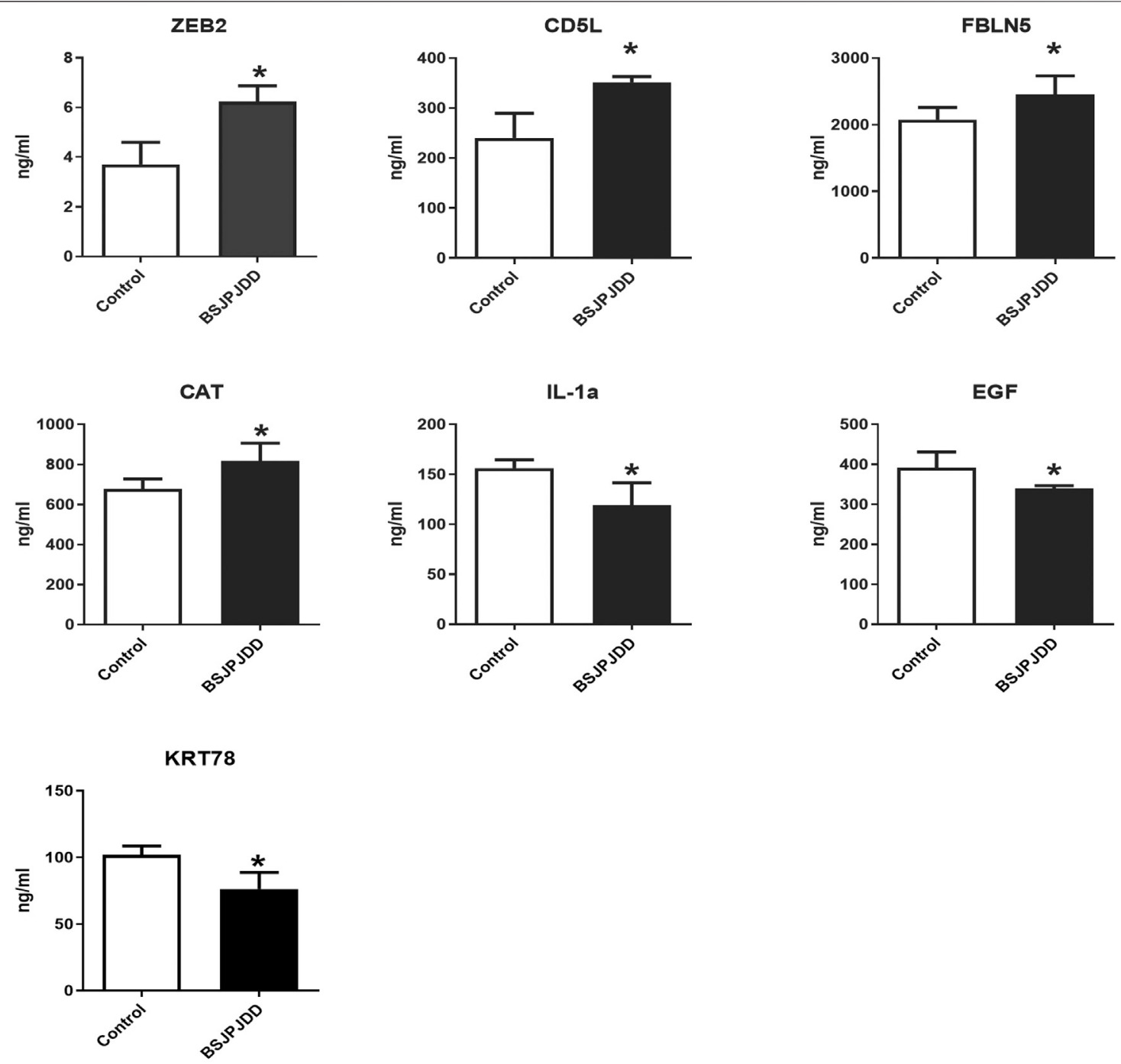

FIGURE 4 | Plasma protein expressions in PCRC patients with LYKD-SDS treated with BSJPJDD $(n=58)$ and without BSJPJDD ( $n=58)$. The validation tests were carried out by ELISA plasma ZEB2,CD5L,FBLN5, and CAT were up regulated,IL-1A, EGF and KRT78 were down-regulated by BSJPJDD * $p<0.05$, BSJPJDD group vs control group.

wighteone, quercetin, and beta-sitosterol) were found in the BSJPJDD, among which quercetin and wighteone targeted IL1A regulating MEKK1, P38, MEK3, TAK1, and isorhamnetin, and wighteone and quercetin targeted EGF regulating JNK and ERK in the MAPK pathway (Figure 3E), indicating that these compounds of BSJPJDD might activate multiple pathways to inhibit tumor progression, increase survival, and improve life quality in PCRC patients.

However, distinguishing inhibitory effects from action effects is difficult in network pharmacology. Moreover, this type of analysis is susceptible to influence by prediction tools. Thus, it is essential to validate the predictions. The cell viability assay is commonly used to evaluate the efficacy of anti-tumor drugs. In this study, $3.84 \mathrm{mg} / \mathrm{mL}$ of BSJPJDD aqueous extract and $15.8 \mu \mathrm{g} / \mathrm{mL}$ of oxaliplatin significantly inhibited the viability of SW620 cells and its CI value was less than 1 (Figure 5). These results showed that there is a synergistic effect of the BSJPJDD aqueous extract and oxaliplatin on inhibiting SW260 cell proliferation.

The MAPK pathway, with three sub-pathways of p38 MAPK, ERK-1/2, and JNK, is an important signaling system that mediates cellular responses, and is involved in many physiological processes, such as cell growth, development, division, and death (Sun et al., 2015). Moreover, it is also an important signaling pathway in the process of the development and death of cancer (Kumar Pandurangan et al., 2018). Recently, it has been reported the MAPK pathway was involved in the regulation of CRC treated by Chinese herbal formulae (Chang et al., 2018). This study found the synergistic effect of BSJPJDD aqueous extract and oxaliplatin. 

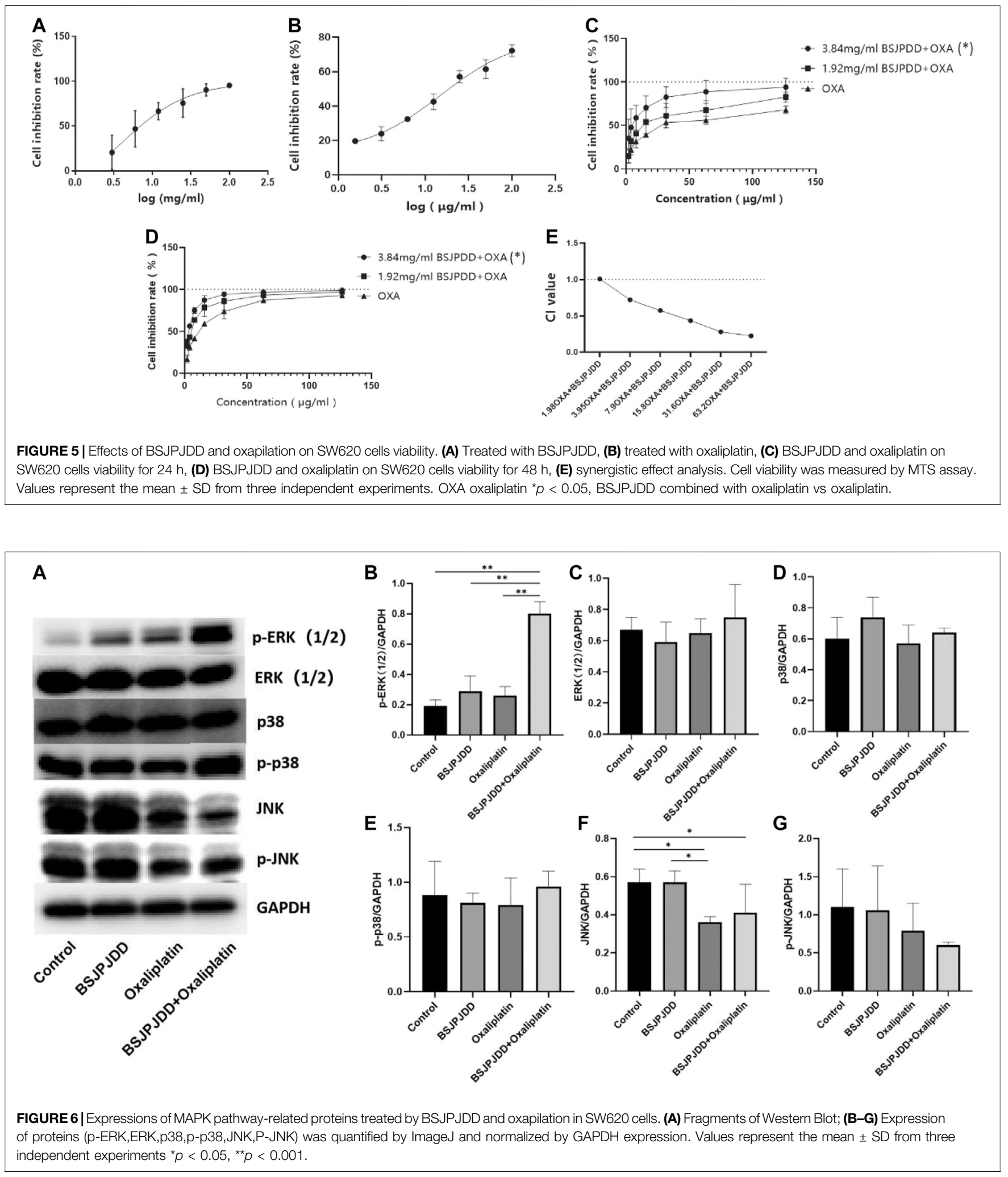


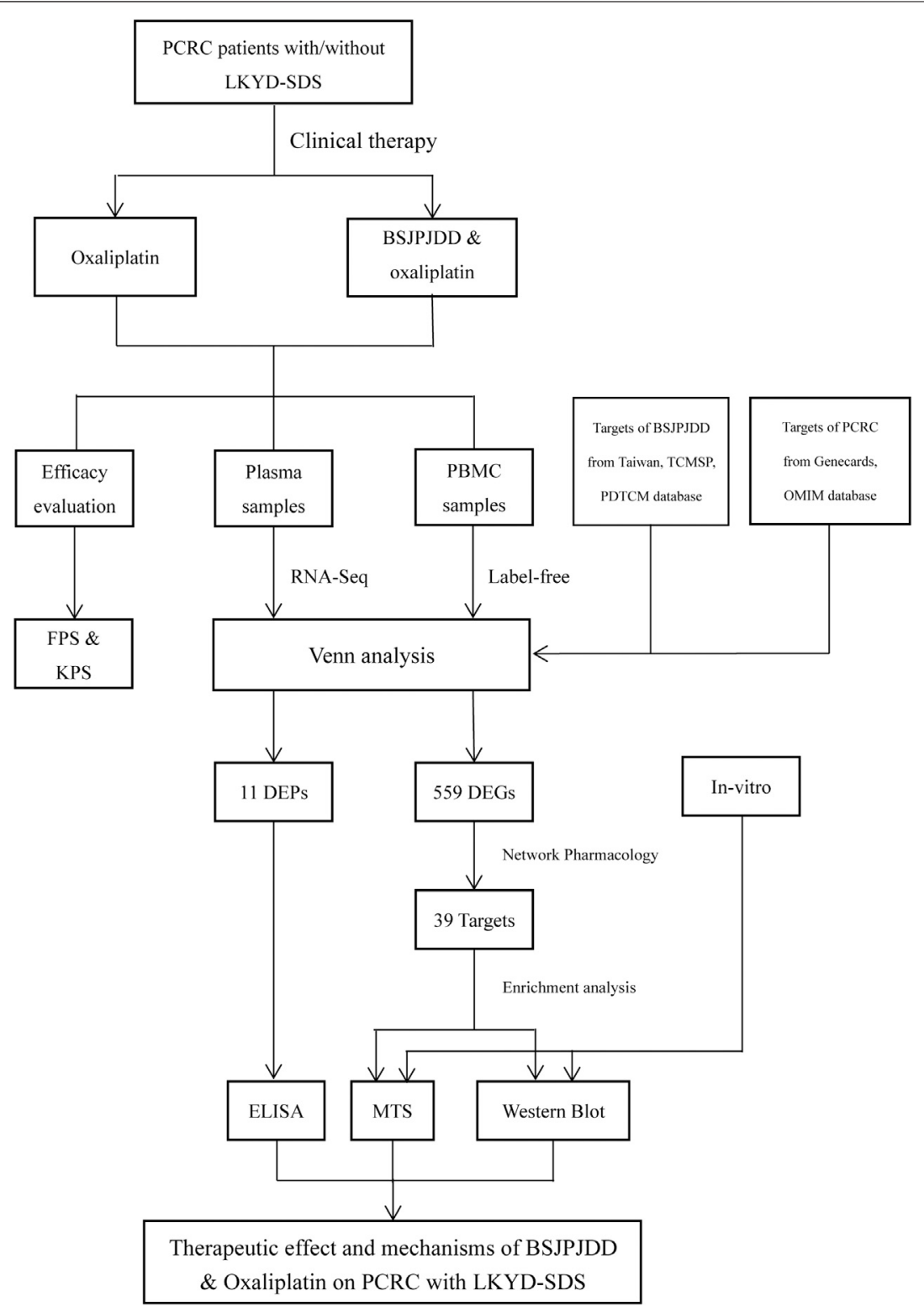

FIGURE 7 | The graphical abstract of the entire circle.

\section{CONCLUSION}

In summary, the clinical efficacy of oxaliplatin including survival time and life quality in PCRC patients with LKYD-SDS were improved by the aqueous extract of BSJPJDD combined with oxaliplatin treatment. Its therapeutic mechanisms may be involved in the comprehensive regulation of BSJPJDD with multi-compounds multi-targets, and multi-efficacies, including the regulation of multiple plasma proteins such as the decreases of ZEB2, CAT, and KRT78 and the increases of IL-1A, CD5L, FBLN5, EGF, and KRT78, and the regulation of multiple signaling pathways such as the downregulation of the MAPK pathway. The graphical abstract of the entire article is shown in Figure 7.

\section{DATA AVAILABILITY STATEMENT}

The datasets presented in this study can be found in online repositories. The names of the repository/repositories and accession number(s) can be found below: NCBI BioProject, accession no: PRJNA682309.

\section{ETHICS STATEMENT}

The studies involving human participants were reviewed and approved by the Institutional Review Board of the Shuguang Hospital affiliated with the Shanghai University of Traditional 
Chinese Medicine. The patients/participants provided their written informed consent to participate in this study.

\section{AUTHOR CONTRIBUTIONS}

M-DY, W-JZ, and JC contributed to the acquisition and analysis of the data and drafting of the paper. W-HW, X-LC, QJ, and QL were responsible for clinical data. M-DY and W-JZ contributed to the experiments in vitro. S-BS and $\mathrm{W}$-HW designed and revised this paper.

\section{REFERENCES}

Barzi, A., Lenz, H.-J., Quinn, D. I., and Sadeghi, S. (2017). Comparative effectiveness of screening strategies for colorectal cancer. Cancer 123 (9), 1516-1527. doi:10.1002/cncr.30518

Binefa, G., Rodríguez-Moranta, F., Teule, A., and Medina-Hayas, M. (2014). Colorectal cancer: from prevention to personalized medicine. WJG 20 (22), 6786-6808. doi:10.3748/wjg.v20.i22.6786

Bos, A. C. R. K., Matthijsen, R. A., Van Erning, F. N., Van Oijen, M. G. H., Rutten, H. J. T., and Lemmens, V. E. P. P. (2018). Treatment and outcome of synchronous colorectal carcinomas: a nationwide study. Ann. Surg. Oncol. 25, 414-421. doi:10.1245/s10434-017-6255-y

Cai, F. F., Zhou, W. J., Wu, R., and Su, S. B. (2018). Systems biology approaches in the study of Chinese herbal formulae. Chin. Med. 13 (1), 65. doi:10.4324/ 9781315505855-5

Chang, J.-S., Lin, H.-J., Deng, J.-S., Wu, W.-T., Huang, S.-S., Huang, G.-J., et al. (2018). Preventive effects of velvet antler (Cervus elaphus) against lipopolysaccharide-induced acute lung injury in mice by inhibiting MAPK/ NF- $\mathrm{kB}$ activation and inducing AMPK/Nrf2 pathways. Evid. Based Complement. Altern. Med. 2018, 1-13. doi:10.1155/2018/2870503

Chen, W., Swanson, B. J., and Frankel, W. L. (2017). Molecular genetics of microsatellite-unstable colorectal cancer for pathologists. Diagn. Pathol. 12 (1), 24. doi:10.1186/s13000-017-0613-8

Chou, T. C., and Talalay, P. (2010). Generalized equations for the analysis of inhibitions of Michaelis-Menten and higher-order kinetic systems with two or more mutually exclusive and nonexclusive inhibitors. Eur. J. Biochem. 115 (1), 207-216. doi:10.1111/j.1432-1033.1981.tb06218.x

Di Costanzo, F., Sobrero, A., Gasperoni, S., Dogliotti, L., Frassineti, L., Falcone, A., et al. (2003). Adjuvant chemotherapy in the treatment of colon cancer: randomized multicenter trial of the italian national intergroup of adjuvant chemotherapy in colon cancer (INTACC). Ann. Oncol. 14, 1365-1372. doi:10. 1093/annonc/mdg359

Gong, B., Kao, Y., Zhang, C., Zhao, H., Sun, F., and Gong, Z. (2019). Exploring the pharmacological mechanism of the herb pair "HuangLian-GanJiang" against colorectal cancer based on network pharmacology. Evid. Based. Complement. Altern. Med. 2019 (2), 1-12. doi:10.1155/2019/2735050

Hong, X. C., Liang, Q. L., Luo, X. B., Hu, K. H., and Zhang, H. J. (2020). Clinical study of Xiangshaliujunzi decoction combined with s-1 as maintenance therapy for stage III or IV gastric carcinoma and colorectal carcinoma. Med. 99 (19), e20081. doi:10.1097/md.0000000000020081

Hu, W., Lei, L., Xie, X., Huang, L., Cui, Q., Dang, T., et al. (2019). Heterogeneous nuclear ribonucleoprotein 1 facilitates recruitment of 53bpl and brcal at the dna break sites induced by oxaliplatin in colorectal cancer. Cell Death Dis. 10 (8), 550. doi:10.1038/s41419-019-1784-x

Hu, X. Q., Wei, B., Song, Y. N., Ji, Q., Li, Q., Luo, Y. Q., et al. (2018). Plasma metabolic profiling on postoperative colorectal cancer patients with different traditional Chinese medicine syndromes. Complement. Ther. Med. 36, 14-19. doi:10.1016/j.ctim.2017.11.006

Huang, S., Peng, W., Mao, D., Zhang, S., Xu, P., Yi, P., et al. (2019). Kangai injection, a traditional Chinese medicine, improves efficacy and reduces toxicity

\section{FUNDING}

This work was supported by key projects of the National Natural Science Foundation of China (No. 81330084) and the Shanghai Baoshan Hospital of Integrated Traditional Chinese and Western Medicine Project (GZRPYJJ-201707).

\section{SUPPLEMENTARY MATERIAL}

The Supplementary Material for this article can be found online at: https:/www.frontiersin.org/articles/10.3389/fphar.2021.524663/ full\#supplementary-material.

of chemotherapy in advanced colorectal cancer patients: a systematic review and meta-analysis. Evid Based. Complement. Altern. Med. 2019 (4), 1-15. doi:10.1155/2019/8423037

Ji, Q., Wang, W., Luo, Y., Cai, F., Lu, Y., Deng, W., et al. (2017). Characteristic proteins in the plasma of postoperative colorectal and liver cancer patients with Yin deficiency of liver-kidney syndrome. Oncotarget 8 (61), 103223-103235. doi:10.18632/oncotarget.21735

Kumar Pandurangan, A., Divya, T., Kumar, K., Dineshbabu, V., Velavan, B., and Sudhandiran, G. (2018). Colorectal carcinogenesis: insights into the cell death and signal transduction pathways: a review. World J. Gastrointest. Oncol. 10 (9), 244-259. doi:10.4251/wjgo.v10.i9.244

Labianca, R., Nordlinger, B., Beretta, G. D., Brouquet, A., and Cervantes, A. (2010). Primary colon cancer: ESMO Clinical Practice Guidelines for diagnosis, adjuvant treatment and follow-up. Ann. Oncol. 21 (Suppl. 5), v70-v77. doi:10.1093/annonc/mdq168

Li, K., Xu, G., Liu, C., Zhu, B., Liu, R., Hua, B.., et al. (2019). Effect of a modified Banxia Xiexin decoction plus chemotherapy on stage III colon cancer. J. Tradit Chin. Med. 39 (2), 251-257. doi:10.19852/j.cnki.jtcm.2019.02.014

Li, P., Li, F., Fang, Y., Wan, D., Pan, Z., Chen, G., et al. (2013). Efficacy, compliance and reasons for refusal of postoperative chemotherapy for elderly patients with colorectal cancer: a retrospective chart review and telephone patient questionnaire. PLos One 8 (2), e55494. doi:10.1371/journal.pone.0055494

Limagne, E., Thibaudin, M., Nuttin, L., Spill, A., Derangère, V., Fumet, J.-D., et al. (2019). Trifluridine/tipiracil plus oxaliplatin improves PD-1 blockade in colorectal cancer by inducing immunogenic cell death and depleting macrophages. Cancer Immunol. Res. 7 (12), 1958-1969. doi:10.1158/23266066.cir-19-0228

Liu, H., Liu, H., Zhou, Z., Parise, R. A., Chu, E., and Schmitz, J. C. (2018a). Herbal formula huang qin ge gen tang enhances 5-fluorouracil antitumor activity through modulation of the E2F1/TS pathway. Cell Commun. Signal. 16 (1), 1-12. doi:10.1186/s12964-018-0218-1

Liu, S.-S., Shi, Q., Li, H.-J., Yang, W., Han, S.-S., Zong, S.-Q., et al. (2017a). Rightand left-sided colorectal cancers respond differently to traditional Chinese medicine. WJG 23, 7618-7625. doi:10.3748/wjg.v23.i42.7618

Liu, X., Wu, J., Zhang, D., Wang, K., Duan, X., and Zhang, X. (2018b). A network pharmacology approach to uncover the multiple mechanisms of hedyotis diffusa willd. on colorectal cancer. Evid. Based Complement Alternat. Med. 2018, 1-12. doi:10.1155/2018/6517034

Liu, Y., Wang, M., Luo, Y., Chen, J., Lu, Y., Shi, Y., et al. (2017b). Mirna-target network analysis identifies potential biomarkers for traditional Chinese medicine (TCM) syndrome development evaluation in hepatitis b caused liver cirrhosis. Sci. Rep. 7 (1), 11054. doi:10.1038/s41598-017-11351-5

Lv, J., Jia, Y., Li, J., Kuai, W., and Li, Z. (2019). Gegen qinlian decoction enhances the effect of pd-1 blockade in colorectal cancer with microsatellite stability by remodelling the gut microbiota and the tumour microenvironment. Cell Death Dis. 10 (6), 415. doi:10.1038/s41419-019-1638-6

Panczyk, M. (2014). Pharmacogenetics research on chemotherapy resistance in colorectal cancer over the last 20 years. World J. Gastroenterol. 20 (29), 9775-9827. doi:10.3748/wjg.v20.i29.9775

Shi, S. H., Cai, Y. P., Cai, X. J., Zheng, X. Y., Cao, D. S., Ye, F. Q., et al. (2014). A networkpharmacology approach to understanding the mechanisms of action of 
traditionalmedicine: bushenhuoxue formula for treatment of chronic kidney disease. PLoS One 9 (3), e89123. doi:10.1371/journal.pone.0089123

Song, Y.-N., Zhang, G.-B., Lu, Y.-Y., Chen, Q.-L., Yang, L., Wang, Z.-T., et al. (2016). Huangqi decoction alleviates dimethylnitrosamine-induced liver fibrosis: an analysis of bile acids metabolic mechanism. J. Ethnopharmacol. 189, 148-156. doi:10.1016/j.jep.2016.05.040

Su, S. B., Lu, A., Li, S., and Jia, W. (2012). Evidence-based Zheng: a traditional Chinese medicine syndrome. Evid. Based Complement. Alternat Med. 2012 (2), 246538. doi:10.1155/2012/246538

Sui, H., Duan, P., Guo, P., Hao, L., Liu, X., Zhang, J., et al. (2017). Zhi Zhen Fang formula reverses Hedgehog pathway mediated multidrug resistance in colorectal cancer. Oncol. Rep. 38, 2087-2095. doi:10.3892/or.2017.5917

Sun, Y., Liu, W. Z., Liu, T., Feng, X., Yang, N., and Zhou, H.-F. (2015). Signaling pathway of MAPK/ERK in cell proliferation, differentiation, migration, senescence and apoptosis. J. Recept. Signal Transduct.Res. 35 (6), 600-604. doi:10.3109/10799893.2015.1030412

Tan, K. Y., Liu, C. B., Chen, A. H., Ding, Y. J., Jin, H. Y., Seow-Choen, F., et al. (2008). The role of traditional Chinese medicine in colorectal cancer treatment. Tech. Coloproctol. 12 (1), 1-6. doi:10.1007/s10151-008-0392-z

Wu, C., Li, M., and Meng, H. (1997). Ministry of health of the people's Republic of China. Guidelines for clinical research on new Chinese medicine series 3. Beijing, China: China Medical Science Press.

Wu, R., Li, X. Y., Wang, W. H., Cai, F. F., Chen., X. L., et al. (2019b). Network pharmacology-based study on the mechanism of bushen-jianpi decoction in liver cancer treatment, Evid. Based Complement. Alternat Med. 2019. 3242989. doi:10.1155/2019/3242989

Wu, R., Dong, S., Cai, F. F., Chen, X. L., Yang, M. D., Liu, P., et al. (2019a). Active compounds derived from fuzheng huayu formula protect hepatic parenchymal cells from apoptosis based on network pharmacology and transcriptomic analysis. Molecules 24 (2), 338. doi:10.3390/molecules 24020338
Yang, M. D., Chen, X. L., Hu, X. Q., Xie, X. Z., and Su, S. B. (2019). Traditional chinese medicine syndromes distribution in colorectal cancer and its association with western medicine treatment and clinical laboratory indicators. World J Tradit Chin Med. 5 (2), 81-87. doi:10.4103/wjtcm. wjtcm_26_18

Ye, L., Jia, Y., Ji, K. E., Sanders, A. J., Xue, K., Ji, J., et al. (2015). Traditional Chinese medicine in the prevention and treatment of cancer and cancer metastasis. Oncol. Lett. 10 (3), 1240-1250. doi:10.3892/ol.2015.3459

Zhang, G. B., Li, Q. Y., Chen, Q. L., and Su, S. B. (2013). Network pharmacology: a new approach for chinese herbal medicine research. Evid. Based Complement. Alternat Med. 2013, 1-9. doi:10.1155/2013/621423

Zhang, S., Shi, L., Mao, D., Peng, W., Sheng, C., Ding, C., et al. (2018). Use of jianpi jiedu herbs in patients with advanced colorectal cancer: a systematic review and meta-analysis. Evid.Based Complementary Altern. Med. 2018, 1-13. doi:10. $1155 / 2018 / 6180810$

Zhu, H., Hao, J., Niu, Y., Liu, D., Chen, D., and Wu, X. (2018). Molecular targets of Chinese herbs: a clinical study of metastatic colorectal cancer based on network pharmacology. Sci. Rep. 8 (1), 1-2. doi:10.1038/s41598-018-25500-x

Conflict of Interest: The authors declare that the research was conducted in the absence of any commercial or financial relationships that could be construed as a potential conflict of interest.

Copyright $\odot 2021$ Yang, Zhou, Chen, Chen, Ji, Li, Wang and Su. This is an openaccess article distributed under the terms of the Creative Commons Attribution License (CC BY). The use, distribution or reproduction in other forums is permitted, provided the original author(s) and the copyright owner(s) are credited and that the original publication in this journal is cited, in accordance with accepted academic practice. No use, distribution or reproduction is permitted which does not comply with these terms. 